\title{
Una aproximación a las funciones ejecutivas en el trastorno del espectro autista
}

\author{
Juan Martos-Pérez, Isabel Paula-Pérez
}

Introducción. La hipótesis psicológica de la disfunción ejecutiva desempeña un papel crucial para explicar el fenotipo conductual de las personas con trastornos del espectro autista (TEA), relacionada también con otras hipótesis como el déficit en teoría de la mente o la hipótesis de la coherencia central débil. Sin embargo, ninguna de estas hipótesis son mutuamente excluyentes y los comportamientos que tienen su origen en alguna de esas tres hipótesis están también moldeados y mantenidos por otros procesos y factores.

Desarrollo. Este artículo revisa la manifestación conductual y el estado de la investigación sobre las funciones ejecutivas en personas con TEA y su impacto en las habilidades de planificación, de flexibilidad mental y cognitiva, generatividad, inhibición de respuesta, habilidades mentalistas y sentido de la actividad.

Conclusión. Aunque la disfunción ejecutiva ha ido ganando peso como hipótesis explicativa en las personas con TEA, persisten algunas dificultades relevantes que precisan de mayor y más detallada investigación. Por otro lado, son muy escasos los programas de intervención con eficacia demostrada que minimicen los efectos de la disfunción ejecutiva en el autismo.

Palabras clave. Generatividad. Flexibilidad mental y cognitiva. Funciones ejecutivas. Habilidades mentalistas. Inhibición de respuesta. Planificación. Sentido de la actividad. Trastornos del espectro autista.

\section{Introducción}

El trastorno del espectro autista (TEA) es el término que se utiliza para dar cuenta de las distintas manifestaciones de gravedad del autismo. Éste, el autismo, es un trastorno del neurodesarrollo que se caracteriza por alteraciones cualitativas en la interacción social recíproca y en la comunicación, y por la presencia de conductas repetitivas e intereses restringidos. Algunos estudios epidemiológicos recientes informan de que el autismo afecta al 0,6\% de la población, es tres veces más frecuente en varones que en mujeres y es habitual su asociación con discapacidad intelectual (cociente intelectual, CI $<70$ ), que está presente en el $25-40 \%$ de los casos [1]. El espectro también incluye a personas de 'alto funcionamiento' o con síndrome de Asperger.

Se han propuesto distintas teorías cognitivas para tratar de entender el funcionamiento psicológico de los TEA; es decir, de la posible relación existente entre su funcionamiento cerebral y su conducta. Una de las más conocidas es la hipótesis del déficit en teoría de la mente [2]. Desde esta hipótesis se propone que ciertos fallos en algunos de los componentes del cerebro social (como la capacidad de atribuir estados mentales y emocionales) están en la base de las dificultades que experimentan las personas con autismo. Aunque esta posición teórica se muestra relativamente potente para dar cuenta y explicar muchas de las conductas que característicamente están asociadas al autismo -en especial las alteraciones sociocomunicativas-, no puede explicar con claridad otras características no sociales del trastorno. Estas características no sociales de los TEA son muy variadas e incluyen un repertorio repetitivo, restrictivo y obsesivo de intereses, inflexibilidad, rigidez y perseveración, así como un patrón disarmónico de inteligencia. Una de las teorías cognitivas que se ha propuesto en los últimos años y que se muestra con éxito para dar cuenta del funcionamiento psicológico en los TEA -incluyendo esas características no sociales- es la teoría de la disfunción ejecutiva. Los grupos de trabajo de Ozonoff y Russell son los mejores exponentes de esta explicación [3,4].

\section{¿Qué son las funciones ejecutivas?}

Las funciones ejecutivas son un constructo, un 'paraguas' bajo el que se cobijan muchas y distintas funciones, como las habilidades de planificación, memoria de trabajo, inhibición y control de impul-
Diagnóstico, Evaluación de Lenguaje y Tratamiento del Espectro Autista, DELETREA; Madrid (J. Martos-Pérez). Departamento de Métodos de Investigación y Diagnóstico en Educación; Institut de Ciències de l'Educació; Universitat de Barcelona; Barcelona, España (I. Paula-Pérez)

Correspondencia:

Dr. Juan Martos Pérez. DELETREA Doctor Esquerdo, 82. E-28007 Madrid.

E-mail:

deletrea@telefonica.net

Aceptado tras revisión externa: 20.01.11.

Cómo citar este artículo: Martos-Pérez J, Paula-Pérez I. Una aproximación a las funciones ejecutivas en el trastorno del espectro autista. Rev Neurol 2011; 52 (Supl 1): S147-53.

(c) 2011 Revista de Neurología 
sos, cambio de foco atencional, flexibilidad, generatividad, iniciación y autorregulación de la acción, entre otras. Recientemente algunos autores [5] prefieren considerar las funciones ejecutivas como un cúmulo de procesos componentes que trabajan conjuntamente más que como un constructo unitario. Aunque puedan existir matizaciones en las distintas definiciones de lo que es función ejecutiva, todas ellas comparten los aspectos centrales que se relacionan con la organización de la acción y el pensamiento. Papazian et al [6] han definido las funciones ejecutivas como 'los procesos mentales mediante los cuales resolvemos deliberadamente problemas internos y externos. Los problemas internos son el resultado de la representación mental de actividades creativas y conflictos de interacción social, comunicativos, afectivos y motivacionales nuevos y repetidos. Los problemas externos son el resultado de la interacción entre el individuo y su entorno. La meta de las funciones ejecutivas es solucionar estos problemas de una manera eficaz y aceptable para la persona y la sociedad. TirapuUstárroz [7], de manera sintética, concibe las funciones ejecutivas como 'un conjunto de procesos cognitivos que actúan en aras de la resolución de situaciones novedosas para las que no tenemos un plan previo de resolución'.

Las funciones ejecutivas, históricamente, se han ubicado en el lóbulo frontal del cerebro, y más específicamente en el córtex prefrontal, donde parece situarse el 'puesto de mando' que organiza y coordina, obviamente, con la amplia interconexión de muchas otras regiones encefálicas (la corteza de asociación posterior, la corteza premotora, los ganglios basales, el tálamo, el hipotálamo, el hipocampo, la amígdala, la corteza cingulada, los núcleos del tallo cerebral y el cerebelo). La adquisición de las funciones ejecutivas se inicia de manera temprana en el desarrollo (aproximadamente desde el año de edad) y se desarrolla lentamente, con dos picos a los 4 y a los 18 años; después se estabiliza y declina en la vejez. El aumento de volumen del córtex prefrontal es lento hasta la edad de 8 años, rápido entre los 8 y los 14 años y, en adelante, se estabiliza hasta adquirir los valores del adulto hacia los 18 años [8]. Las funciones ejecutivas están alteradas en pacientes con daño adquirido en los lóbulos frontales y en un amplio rango de trastornos del desarrollo que se relacionan con déficit congénitos en los lóbulos frontales. Entre tales trastornos se incluyen el trastorno por déficit de atención/hiperactividad (TDAH), el TEA, el trastorno obsesivo-compulsivo, el síndrome de Tourette y la esquizofrenia [9]. La investigación con técnicas de neuroimagen ha mostrado un desa- rrollo anormal de estructuras del lóbulo frontal [10], menor densidad de materia gris y anormalidades en el funcionamiento en regiones frontoestriatales [11, 12] en los TEA.

Aunque el constructo de funciones ejecutivas no esté universalmente consensuado, lo que sí está claro es que una disfunción ejecutiva dificulta a la persona llevar una vida independiente y tener un comportamiento consistente, pues afecta a funciones de orden superior como la toma de decisiones, las habilidades mentalistas, la resolución de problemas, la regulación emocional, la generalización de los aprendizajes, la adaptación a situaciones imprevistas y novedosas, etc., que son indispensables para funcionar de manera socialmente adaptada.

La primera vez que surge un estudio empírico que correlaciona funciones ejecutivas y TEA fue en 1991, de la mano de Ozonoff et al [13]. En sus trabajos demuestran que las medidas ejecutivas son iguales o más eficaces que las pruebas en teoría de la mente para discriminar grupos de sujetos autistas de grupos de sujetos control; sin embargo, cuando revisamos los estudios de función ejecutiva en autismo, emerge una imagen general algo borrosa, que no es suficientemente clara, y una de las razones de ello se debe a que una gran parte de los estudios se ha realizado con niños y adolescentes con distintas edades y CI por debajo del rango normal. Por el contrario, los estudios con muestras de adultos han utilizado sujetos con CI dentro del rango normal o superior. Otra de las razones se debe a los también distintos grupos de control utilizados, que no siempre son los más apropiados para comparar con personas con autismo.

\section{Planificación}

La planificación es una operación compleja y dinámica en la que una secuencia de acciones planificadas se debe monitorizar, reevaluar y actualizar constantemente [14]. Las tareas más típicamente utilizadas son la torre de Hanoi y la torre de Londres. Las personas con autismo (niños, adolescentes y adultos) presentan déficit de planificación comparados con personas de desarrollo normal y con personas con otros trastornos que no se asocian a discapacidad intelectual. Cuando se comparan adultos con autismo y dificultades de aprendizaje y sujetos con dificultades de aprendizaje pero fuera del espectro, aparecen diferencias: las personas con autismo rinden peor en habilidades de planificación y memoria de trabajo [15]. No obstante, conviene tener cautela a la hora de interpretar los 
resultados, porque en autismo, dados los niveles de inteligencia tan heterogéneos, no está suficientemente claro si los déficit de planificación identificados reflejan un efecto del autismo en todos los individuos o de la discapacidad intelectual de algunos de ellos. Por otro lado, algún estudio [16] que usa versiones computarizadas de las tareas anteriores (y otras tareas) ha mostrado que las dificultades de planificación no aparecen en todas las situaciones, sino en aquellas que implican un mayor nivel de complejidad, lo que puede explicar mejor las dificultades con que se encuentran las personas con autismo para desenvolverse en la vida cotidiana. Las tareas utilizadas para evaluar la planificación, como la torre de Hanoi/torre de Londres, también implican la utilización de otros procesos cognitivos (memoria de trabajo, inhibición de respuestas prepotentes) y además, cuando se han utilizado en la población normal, se observa una baja fiabilidad test-retest [17], por lo que conviene actuar con prudencia.

\section{Flexibilidad cognitiva}

La flexibilidad cognitiva, también denominada 'cambio de criterio', es otra de las funciones ejecutivas evaluadas. Estos términos hacen referencia a la habilidad para cambiar a un pensamiento o acción diferente en función de los cambios que ocurren en las situaciones o contextos. Las estereotipias, la rigidez e inflexibilidad cognitiva y conductual, la insistencia en la invarianza ambiental, los rituales y rutinas, las ecolalias tan propios de la sintomatología de los TEA son los indicadores conductuales más íntimamente relacionados con las funciones ejecutivas. Sin embargo, no es una sintomatología específica y única de las personas con TEA, ya que aparece en otros cuadros clínicos como, por ejemplo, el retraso mental, la esquizofrenia, o la discapacidad sensorial.

Una flexibilidad cognitiva pobre se caracteriza también por dificultades en la regulación y modulación de los actos motores. La ejecución de personas con TEA en tareas de clasificación de cartas de Wisconsin ha mostrado un mayor nivel de respuestas perseverativas de error, que se atribuyen a un déficit en flexibilidad mental, en comparación con el desarrollo normal y otros grupos con diferentes trastornos (TDAH, trastornos específicos en el lenguaje y dislexia). Algunos estudios con pacientes con lesiones en el lóbulo frontal [18] están informando de que el perfil de errores perseverativos varía en función de la ubicación de la lesión. Una ma- yor profundización en estos hallazgos, así como en la clarificación de otros factores (¿ocurren los errores de perseveración en todo el espectro?, ¿influye el CI?, ¿la conducta perseverativa se relaciona directamente con la conducta repetitiva y estereotipada en la vida diaria?), contribuirá a esclarecer el funcionamiento de las personas con autismo en la función ejecutiva de flexibilidad cognitiva.

Una alteración más o menos grave de las funciones ejecutivas, relacionada con la flexibilidad cognitiva y conductual, es la capacidad generativa, la cual provoca alteraciones en la capacidad para generar objetivos y metas adecuadas y dificultad para generar nuevos comportamientos. Esto conduce, inexorablemente, a una limitación de la creatividad, de la espontaneidad, de la flexibilidad y de la adaptación a los entornos, de la generación de planes de acción y, en el peor de los casos, a una repetición continua de la conducta o a un empleo estereotipado de los objetos, juegos y uso de los tiempos de ocio. Se trata de un proceso mental de generación de esquemas cognitivos nuevos que se adaptan y se asimilan a los anteriormente aprendidos y que, cuando se deteriora, provoca que las situaciones nuevas se vivan como 'terroríficamente novedosas'. Ello explicaría el deseo de invarianza ambiental y el rechazo a situaciones no conocidas o imprevistas, que convierte el comportamiento de la persona con autismo en muy predecible y carente de iniciativa y creatividad. Recientemente, se ha puesto en relación con los inusuales patrones de habilidades de memoria (por ejemplo, una dificultad en la memoria episódica) para explicar las dificultades en generatividad y, en general, en la imaginación y la planificación del día a día [19].

Turner [20] ha propuesto que las dificultades en la capacidad para generar espontáneamente nuevas ideas y conductas están en la base de la ausencia de espontaneidad e iniciativa, la pobreza de habla y acción y los fallos en la actividad simbólica e imaginativa que caracterizan al autismo. El trabajo que ha desarrollado esta autora se fundamenta sobre dos hipótesis relacionadas y complementarias. En la primera se plantea que una alteración en la capacidad de controlar la inhibición del comportamiento puede comportar la repetición de acciones y pensamientos debido a que la persona -incapaz de regular la atención y la acción de manera normal- se ve abocada a seguir una única línea de conducta. En la segunda hipótesis se establece que la incapacidad para producir conductas nuevas de forma autogenerada se puede manifestar como repetición de comportamientos de un repertorio conductual reducido y bien ensayado. 


\section{Inhibición de respuesta}

La inhibición de acciones no deseadas que se están ejecutando o la inhibición de comportamientos prepotentes son uno de los procesos mentales imprescindibles para la regulación y el control del comportamiento, su flexibilidad y adaptación. La alteración de dichos procesos de inhibición puede convertirse en el sustrato psicológico del comportamiento repetitivo y estereotipado, y el deseo de invarianza de las personas con TEA. Si la persona no es capaz de inhibir pensamientos o acciones antes o durante el ejercicio de una actividad, tenderá a la perseveración, la rigidez y la persistencia, como si no fuera dueña de su capacidad para parar y dirigir su conducta en otra dirección. La inhibición también se ha estudiado en el autismo, y no se han encontrado diferencias en la ejecución cuando se compara con el desarrollo normal usando tareas prototípicas de interferencia como la tarea de Stroop (lo que constituye un contraste con otras alteraciones del desarrollo como el TDAH [9]) y las tareas de go/no go y de priming negativo). El único tipo de inhibición en el que algunos estudios han observado fallos en el autismo es la inhibición de respuesta prepotente [13]. No obstante, se precisan nuevos estudios que clarifiquen si las dificultades en inhibición de respuesta prepotente pueden estar más bien relacionadas con el nivel de habilidad e inteligencia de las personas con autismo. El nivel de gravedad de los procesos de inhibición conductual predecirá que la perseveración pueda diferenciarse entre:

- Perseveración de respuesta simple, repitiendo la misma secuencia de conducta, con repetición de conductas de bajo nivel (por ejemplo, movimientos estereotipados, manipulación estereotipada de objetos, etc.).

- Perseveración de comportamientos de alto nivel, con secuencias de acción variables en torno a un tema que nunca cambia (por ejemplo, intereses limitados, adhesión rígida a rutinas y rituales, lenguaje repetitivo, etc.) [21].

En líneas generales, son relativamente inconsistentes los hallazgos en relación con la conducta repetitiva, con datos a favor y en contra. Mientras algunos autores encuentran, en niños, una floja relación entre la presencia de conductas repetitivas y medidas concretas de función ejecutiva [22], otros han hallado (aplicando una batería extensa de tareas de función ejecutiva) una correlación con una medida combinada de conducta repetitiva basada en la entrevista a los padres y la observación clínica [23]. Una línea de investigación prometedora es la que está estableciendo relación entre las dificultades con el ajuste y cambio de criterio cognitivo y los intereses repetitivos [23,24].

\section{Habilidades mentalistas}

Una de las funciones superiores del ser humano más fascinantes es la de ser capaces de atribuir estados mentales y emocionales a los demás, poder anticipar sus comportamientos, sus intenciones, intuir o saber aquello que los motiva; en definitiva, leer sus mentes. Esta habilidad mentalista es imprescindible para sobrevivir en el mundo social, para relacionarnos, tener amigos, convivir con la familia, coordinarnos con otras personas, cooperar con otros, engañarlos, enfrentarnos a ellos, competir, disfrutar de una relación, etc. Y para ello hemos de ser capaces de desarrollar un proceso ejecutivo: poseer un plan propio, conocer o intuir los planes que tienen los otros en su mente formándonos una representación mental de ello, y considerar las consecuencias de ambos planes, los propios y los ajenos. De ello dependerá el éxito de la resolución en el desempeño de la interacción social. Es por ello que, dentro de los TEA de alto funcionamiento cognitivo, podemos encontrar a personas muy inteligentes en alguna área concreta (matemáticas, música, física, etc.) y, sin embargo, muy torpes en el área de las relaciones sociales.

Las habilidades mentalistas requieren de procesos cognitivos relacionados con las funciones ejecutivas, de manera que no pueden atribuirse únicamente a la inferencia de los estados mentales y emocionales de los demás. Clásicamente, la teoría de la mente se evalúa con tareas de falsa creencia, pero el éxito o el fracaso en su ejecución van más allá de la capacidad intersubjetiva de atribuir estados mentales y emocionales a los otros, ya que también están en juego habilidades ejecutivas como, por ejemplo, la inhibición de una respuesta factual prepotente (el objeto cambiado de lugar), la generación de pensamiento no preestablecido por la información (la atribución de creencia), la memoria de trabajo (mantener la información simultánea acerca de la acción de los personajes, las diferencias de perspectiva de cada uno, y entre lo que ven, creen y saben), la secuenciación en pasos sucesivos, etc. [25].

Cuando se analiza la capacidad de mentalización en los TEA, desde el punto de vista del funcionamiento ejecutivo, nos encontramos con tres posicionamientos [14]. En el primero se argumenta que es el desarrollo de las funciones ejecutivas lo que permite que se desarrolle la teoría de la mente en el 
niño [3,4]. En el segundo se propone que no hay sistemas específicos para procesar estados mentales y que la ejecución en tareas de teoría de la mente se puede explicar desde la habilidad en la función ejecutiva [25]; por ejemplo, una ejecución pobre en la tarea de falsa creencia puede estar indicando simplemente que hay un déficit en la habilidad para inhibir una respuesta prepotente al mismo tiempo que debe mantenerse en la memoria de trabajo una información de acción relevante. Las habilidades mentalistas requieren de procesos cognitivos relacionados con las funciones ejecutivas y su desempeño. Como decíamos, no puede atribuirse únicamente a la inferencia de los estados mentales y emocionales de los demás [26]. Un tercer posicionamiento adopta el punto de vista contrario al argumentar que la capacidad para representar estados mentales es necesaria para el desarrollo de la función ejecutiva [27]. Desde esta perspectiva, una persona debe ser capaz de representarse sus propias intenciones en el establecimiento de un plan.

\section{Sentido de la actividad}

Una de las características más relevantes de las personas es que somos seres propositivos, encarados al futuro, ya sea a corto, medio o largo plazo (planificamos permanentemente: lo que haremos por la tarde o lo que queremos ser de mayores), y, además, somos seres intencionales. Nada de lo que hacemos deja de tener un sentido, por muy sencillo y simple que sea. Y para guiar dicho comportamiento prospectivo necesitamos representarnos las imágenes mentales del futuro, visualizar mentalmente aquello que nos proponemos, anticipando y previendo cómo se sucederán los acontecimientos. Fijamos metas y objetivos y después actuamos conforme a lo que nos proponemos. Para ello, se precisa de capacidades de organización y planificación que serán realmente complejas según la finalidad de la acción; por ejemplo: hacer la compra, preparar un viaje, obtener un título universitario, etc., requieren de diferentes niveles organizativos, previsibilidad o anticipación.

La investigación sobre el sentido de la actividad en los TEA desde la óptica de las funciones ejecutivas, en cuanto a la dificultad que se observa para dar sentido a la acción propia [28], es escasa. En los casos de mayor afectación se traduce en un vacío de acción funcional y, en los de mejor nivel, en una dificultad para proyectarse en el futuro. La falta de sentido se relaciona con la inflexibilidad, la dificultad para aceptar cambios y las limitaciones en las competencias de anticipación. Por otro lado, esta dificultad para dar sentido a la acción propia se conecta claramente con la percepción fragmentada de la realidad [29], con la teoría de la coherencia central débil y con una percepción basada más en los detalles y procesos de representación visuoespacial simultánea que en secuencias temporales. La carencia de esquemas o dificultad para organizar secuencias activas en función de metas anticipadas se traduce en la emisión de acciones sin propósito.

Además, el sentido se relaciona con el contexto y con la habilidad para desambiguarlo. La prospectiva de la investigación respecto a las funciones ejecutivas y los TEA se sitúa en la habilidad para resolver la ambigüedad o desambiguar las situaciones. Si me piden mi Documento Nacional de Identidad para hacer una gestión, la respuesta es única, no hay elección. Ese documento es el que es y la respuesta es intrínsecamente correcta en esa situación. Cualquier otra respuesta será falsa. En cambio, si me preguntan qué voy a hacer el fin de semana, la respuesta no está determinada, es una respuesta ambigua que implica una toma de decisiones. Conlleva un fuerte nivel de complejidad ejecutiva que no puede solucionarse con una agenda o una calculadora. Se trata de situaciones donde hemos de elegir. En palabras de Goldberg [30]: 'Un individuo debe tener flexibilidad para adoptar diferentes perspectivas sobre la misma situación en diferentes momentos. El organismo debe ser capaz de desambiguar la misma situación de múltiples formas y tener la capacidad de cambiar entre ellas a voluntad. Tratar con la ambigüedad inherente está entre las funciones principales de los lóbulos frontales'

\section{Conclusiones}

A lo largo de las últimas dos décadas, la investigación ha ido confirmando la afectación de las funciones ejecutivas en la población clínica con TEA. La explicación de una disfunción ejecutiva en el autismo ha ido ganando peso gradualmente; sin embargo, persisten algunos problemas sin resolver. Uno de los más relevantes es la falta de consenso acerca de qué aspectos de las funciones ejecutivas están alterados en autismo. Por otro lado, y como ya hemos señalado, resulta un problema el rango de edad y la habilidad de las muestras evaluadas. Una dificultad especial es el hecho de que los fallos de disfunción ejecutiva también se encuentran en otros trastornos. Esta anterior dificultad limita claramente el potencial del uso de la disfunción ejecutiva como un marcador diagnóstico en el autismo, 
por lo que se precisan estudios más detallados del funcionamiento ejecutivo en diferentes poblaciones clínicas. Una última dificultad se relaciona con el hecho de que los fallos en función ejecutiva no se dan de manera universal en el autismo en tanto y en cuanto algunas personas con $\mathrm{CI}$ en el rango normal resuelven de manera similar a los controles algunas tareas concretas de función ejecutiva. Conviene señalar, no obstante, algunas otras posibles y concretas implicaciones que pueden tener una gran relevancia en la investigación futura. Una de ellas se refiere a la conducta repetitiva, de la que se ha sugerido que puede ser un factor altamente heredable en el autismo y que es diferente de los déficit en la interacción social [31]. La otra es la relación que podría determinarse entre los síntomas no sociales y la habilidad para establecer, fijar o realizar el cambio de criterio como fenotipo intermedio potencialmente informativo para vincular los planos psicológico y neurobiológico [24].

Desde el punto de vista de las implicaciones terapéuticas y de tratamiento, como consecuencia de la disfunción ejecutiva se pueden ver especialmente deterioradas las habilidades adaptativas de la vida cotidiana [32,33]. Sin embargo, a pesar de reconocerse la importancia de las funciones ejecutivas en el fenotipo autista, son limitados los programas de intervención comercializados y, de los existentes, son pocos los que han estudiado su eficacia. Una de las excepciones es el estudio de Mesibov [34] sobre la efectividad del sistema TEACCH (Treatment and Education of Autistic and Communication Related Handicapped Children), elaborado por Eric Schopler y aplicado por el Gobierno Federal de Carolina del Norte (Estados Unidos) desde 1966. El sistema TEACCH propone una organización del entorno y una presentación de las actividades que compensan las disfunciones ejecutivas más significativas de los TEA y promueven la acción independiente. Para ello utiliza sistemas visuales y provee rutinas predecibles y altamente estructuradas. Además, rescata las fortalezas e intereses de las personas con TEA, que, por supuesto, también existen. Los datos obtenidos de la investigación confirman que el programa TEACCH permite un gran nivel de independencia y una mayor productividad de las personas con TEA, además de contener protocolos que se pueden implementar fácilmente en una gran variedad de entornos, incluyendo la escuela, el trabajo, el hogar y la comunidad.

También se ha sugerido que el programa denominado REHABIT [35] puede ser eficaz para niños con autismo. Dicho programa está diseñado para enseñar una variedad de habilidades cognitivas, in- cluidas funciones ejecutivas, atención, memoria, lenguaje y rendimiento escolar.

Resulta, pues, necesario realizar acomodaciones, modificaciones y desarrollar habilidades compensatorias que, respetando 'la cultura del autismo', permitan a las personas con TEA minimizar los efectos negativos que la disfunción ejecutiva puede ocasionar en las mil y una situaciones a las que se deben enfrentar en la vida cotidiana y en los contextos de aprendizaje formal y menos formal.

\section{Bibliografía}

1. Chakrabarti S, Fombonne E. Pervasive developmental disorders in preschool children. J Am Med Assoc 2001; 285: 3093-9.

2. Baron-Cohen S, Tager-Flusberg H, Cohen DJ. Understanding others minds. Perspectives from developmental cognitive neuroscience. Oxford: Oxford University Press; 2000.

3. Russell J. El autismo como trastorno de la función ejecutiva. Madrid: Editorial Médica Panamericana; 2000.

4. Ozonoff S. Componentes de la función ejecutiva en el autismo y otros trastornos. In Russell J, ed. El autismo como trastorno de la función ejecutiva. Madrid: Editorial Médica Panamericana; 2000. p. 177-201.

5. Ozonoff S, South M, Provencal S. Executive functions. In Volkmar FR, Paul R, Klin A, Cohen D, eds. Handbook of autism and developmental disorders. New York: Wiley; 2005. p. 606-27.

6. Papazian O, Alfonso I, Luzondo RJ. Trastornos de las funciones ejecutivas. Rev Neurol 2006; 42 (Supl 3): S45-50.

7. Tirapu-Ustárroz J. Estimulación y rehabilitación de las funciones ejecutivas. Módulo didáctico 6. Asignatura 'Estimulación Cognitiva'. Universitat Oberta de Catalunya; 2010.

8. Diamond A. Normal development of prefrontal cortex from birth to young adulthood: cognitive function, anatomy, and biochemistry. In Stuss DT, Knight RT, eds. Principles of frontal lobe function. London: Oxford University Press; 2002. p. 466-503.

9. Ozonoff S, Jensen J. Brief report: specific executive function profiles in three neurodevelopmental disorders. J Autism Dev Disord 1999; 29: 171-7.

10. Carper RA, Courchesne E. Localized enlargement of the frontal cortex in early autism. Biol Psychiatry 2005; 57: 126-33.

11. McAlonan GM, Cheung V, Cheung C, Suckling J, Lam GY, Tai KS, et al. Mapping the brain in autism. A voxel-based MRI study of volumetric differences and intercorrelations in autism. Brain 2005; 128: 268-76.

12. Schmitz N, Rubia K, Daly E, Smith A, Williams S, Murphy DG. Neural correlates of executive function in autistic spectrum disorders. Biol Psychiatry 2006; 59: 7-16.

13. Ozonoff S, Pennington BF, Rogers SJ. Executive function deficits in high-functioning autistic individuals: relationship to theory of mind. J Child Psychol Psychiatry 1991; 32: 1081-105.

14. Hill EL. Evaluation the theory of executive dysfunction in autism. Developmental Review 2004; 24: 189-233.

15. Barnard L, Muldoon K, Hasan R, O'Brien G, Stewart M. Profiling executive dysfunction in adults with autism and comorbid learning disability. Autism 2008; 12: 125-41.

16. Hughes C, Russell J, Robbins TW. Evidence for executive dysfunction in autism. Neuropsychologia 1994; 32: 477-92.

17. Bishop DV, Aamodt-Leeper G, Creswell C, McGurk R, Skuse DH. Individual differences in cognitive planning on Tower of Hanoi task: neuropsychological maturity or measurement error? J Child Psychol Psychiatry 2001; 42: 551-6.

18. Stuss DT, Levine B, Alexander MP, Hong J, Palumbo C, Hamer L, et al. Wisconsin Card Sorting Test performance in patients with focal frontal and posterior brain damage: effects of lesion location and test structure on separable cognitive processes. Neuropsychologia 2000; 38: 388-402. 
19. Boucher J. Memory and generativity in very high functioning autism: a firsthand account and an interpretation. Autism 2007; 11: 255-64.

20. Turner M. Hacia una explicación de la conducta repetitiva en el autismo basada en la disfunción ejecutiva. In Russell J, ed. El autismo como trastorno de la función ejecutiva. Madrid: Editorial Médica Panamericana; 2000. p. 55-98.

21. Hughes C, Russell J. Autistic children's difficulty with mental disengagement from an object. Its implications for theories in autism. Dev Psychol 1993; 29: 498-510.

22. Joseph RM, Tager-Flusberg H. The relationship of theory of mind and executive functions to symptom type and severity in children with autism. Dev Psychopathol 2004, 16: 137-55.

23. López BR, Lincoln AJ, Ozonoff S, Laiz Z. Examining the relationship between executive functions and restricted repetitive symptoms of autistic disorders. J Autism Dev Disord 2005; 35: 445-60.

24. Yerys BE, Wallace GL, Harrison B, Celano MJ, Giedd JN, Kenworthy LE. Set-shifting in children with autism spectrum disorders: reversal shifting deficits on the Intradimensional/ Extradimensional Shift Test correlate with repetitive behaviors Autism 2009; 13: 523-38.

25. Frye D, Zelazo PD, Palfai T. Theory of mind and rule-based reasoning. Cogn Dev 1995; 10: 483-527.

26. Ibáñez-Barassi AM. Autismo, funciones ejecutivas y mentalismo reconsiderando la heurística de descomposición modular. Revista Argentina de Neuropsicología 2005; 6: 25-49.
27. Perner J, Lang B. Theory of mind and executive function: is there a developmental relationship? In Baron-Cohen S, TagerFlugsber H, Cohen D, eds. Understanding others minds: perspectives from developmental cognitive neuroscience. Oxford: Oxford University Press; 2000. p. 150-81.

28. Rivière A. Tratamiento y definición del espectro autista. II. Anticipación; flexibilidad y capacidades simbólicas. In Rivière A, Martos J, eds. El tratamiento del autismo. Nuevas perspectivas. Madrid: Apna-Imserso; 1997. p. 107-60.

29. Frith U. Autismo. Hacia una explicación del enigma. Madrid: Alianza; 1991.

30. Goldberg E. El cerebro ejecutivo. Lóbulos frontales y mente civilizada. Barcelona: Crítica-Drakontos; 2002.

31. Ronald A, Happe F, Plomin R. Genetic research into autism. Science 2006; 311: 952.

32. Clark C, Prior M, Kinsella G. The relationship between executive function abilities adaptive behavior, and academia achievement in children with externalizing behavior problems. J Child Psychol Psychiatry 2002; 43: 785-96.

33. Gilotty L, Kenworthy L, Sirian L, Black D, Wagner AE. Adaptive skills and executive function in autism spectrum disorders. Child Neuropsychol 2002, 8: 241-8.

34. Mesibov GB. Formal and informal measures on the effectiveness of the TEACCH Programme. Autism 1997; 1: 25-35.

35. Jepsen RH, Von Thaden K. The effect of cognitive education on the performance of students with neurological developmental disabilities. Neurorehabilitation 2002; 17: 201-9.

\section{An approach to the executive functions in autism spectrum disorder}

Introduction. The psychological hypothesis of executive dysfunction plays a crucial role in explaining the behavioural phenotype of persons with autism spectrum disorders (ASD), along with other hypotheses such as the deficit in the theory of mind or the weak central coherence hypothesis. Yet, none of these hypotheses are mutually exclusive and behaviours that have their origins in one of these three hypotheses are also shaped and upheld by other processes and factors.

Development. This article reviews the behavioural manifestation and current state of research on the executive functions in persons with ASD. It also examines its impact on planning, mental flexibility and cognitive skills, generativity, response inhibition, mentalist skills and sense of activity.

Conclusions. Although executive dysfunction has become more significant as a hypothesis explaining persons with ASD, there remain some important difficulties in need of further, more detailed research. Moreover, very few intervention programmes have been proved to be effective in minimising the effects of executive dysfunction in autism.

Key words. Autism spectrum disorders. Executive functions. Generativity. Mental and cognitive flexibility. Mental skills. Planification. Response inhibition. Sense of activity. 